\title{
Assessment of plasma microRNAs in congenital intestinal malrotation
}

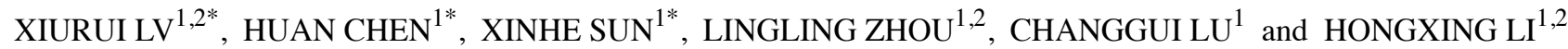 \\ ${ }^{1}$ Department of Pediatric Surgery, Children's Hospital of Nanjing Medical University, Nanjing, Jiangsu 210008; \\ ${ }^{2}$ State Key Laboratory of Reproductive Medicine, Institute of Toxicology, School of Public Health, \\ Nanjing Medical University, Nanjing, Jiangsu 211166, P.R. China
}

Received November 17, 2019; Accepted July 20, 2020

DOI: $10.3892 / \mathrm{mmr} .2020 .11395$

\begin{abstract}
Intestinal malrotation in newborns often requires urgent surgical treatment, especially in the presence of volvulus. Therefore, early-stage diagnosis is critical. In the present study, differentially expressed plasma microRNAs (miRNAs) were screened for in patients with intestinal malrotation using high-throughput Illumina sequencing, and validated using reverse transcription-quantitative PCR. Receiver operating characteristic curve (ROC) analysis was conducted to evaluate their specificity, sensitivity and assess their diagnostic value for intestinal malrotation. Bioinformatics analysis was performed to investigate the functions associated with the dysregulated miRNAs. A profile consisting of 28 differentially expressed plasma miRNAs was obtained, of which nine were verified to exhibit significantly altered expression. According to a ROC analysis, four of these could represent novel early-stage, non-invasive biomarkers for intestinal malrotation. Bioinformatics analysis demonstrated that the differentially expressed miRNAs were predominantly involved in 'metal ion transmembrane transporter activity' and 'calcium-dependent protein binding', which may be related to the 'endocytosis' pathway. In conclusion, significantly differentially expressed plasma miRNAs were identified in congenital intestinal malrotation and their potential roles were described. These differentially expressed miRNAs may serve as biomarkers of intestinal malrotation and improve early diagnosis for this condition.
\end{abstract}

Correspondence to: $\mathrm{Dr}$ Changgui Lu or Dr Hongxing Li, Department of Pediatric Surgery, Children's Hospital of Nanjing Medical University, 72 Guangzhou Road, Nanjing, Jiangsu 210008, P.R. China

E-mail: luchanggui1984@163.com

E-mail: hx8817@126.com

*Contributed equally

Key words: plasma microRNA, intestinal malrotation, biomarker

\section{Introduction}

Intestinal malrotation is a congenital aberrant positioning of the bowel that is usually accompanied with anomalous bowel fixation through mesenteric bands or a lack of fixation in parts of the bowel (1). Intestinal malrotation was first described by William Ladd in 1936 and later classified as non-rotation, mixed or incomplete rotation, reversed rotation, or mesocolic hernia (2,3). Typically, $\leq$ one in 200 newborns are estimated to have asymptomatic rotational anomaly $(3,4)$. Symptomatic malrotation affects 1 in 6,000 live births and often occurs in the first few weeks of life, resulting in elevated risk of bowel obstruction, midgut volvulus and necrosis in the bowel $(3,5,6)$. Currently, upper gastrointestinal (UGI) series is the preferred imaging technique for malrotation diagnosis. Nevertheless, both false positive and false negative interpretations occur, with reported false positive rates of $\leq 15 \%(1,7,8)$. When the duodenojejunal junction position is ambiguous based on UGI series, further examination is necessary. A barium enema might prove helpful in such cases; however, the first pass of barium through the duodenum is often overlooked (9). The duodenal anatomy is hard to recognize with inadequate amounts of barium, while excessive barium could cause rapid and uncontrolled passage of barium through the duodenum or vague visualization. Moreover, the use of sonography in malrotation diagnosis often requires the opinion of experienced radiologists, who may not always available at most centers $(10,11)$. Therefore, the diagnosis of intestinal malrotation is often an intricate and complex process, and a more effective and ideally non-invasive diagnostic method is needed $(6,11,12)$.

MicroRNAs (miRNAs) are a group of non-coding RNA molecules, usually 19-25 nucleotides long, which are abundantly and stably expressed in plasma and other bodily fluids $(13,14)$. Differential expression of specific miRNAs has been identified in numerous diseases, and an increasing body of evidence indicates that miRNAs could serve as promising biomarkers for diagnostic use (15-17). For example, previous studies have identified miRNAs associated with various types of cancer, highlighting an opportunity to use these circulating disease-related miRNAs as biomarkers $(18,19)$. In cardiovascular diseases, it was demonstrated that the combined use of multiple miRNAs could improve the predictive efficiency of traditional diagnostic methods $(20,21)$. Thus, understanding the potential role of miRNAs in intestinal malrotation 
may provide invaluable insight into improved diagnostic approaches, as demonstrated in other diseases (22-24).

Previously, a mutation in the forkhead box F1 gene was demonstrated to be associated with intestinal malrotation (25). However, to the best of the authors' knowledge, no studies have evaluated epigenetic changes during the pathogenesis of intestinal malrotation (26). In the present study, it was hypothesized that the expression of specific miRNAs might be dysregulated in newborns with intestinal malrotation. Thus, the aim of the present study was to determine whether such differentially expressed miRNAs could be used for improved diagnosis of intestinal malrotation.

\section{Materials and methods}

Study design and sample collection. Ten children with intestinal malrotation (age range, 5 days to month) and ten sex- and age-matched controls (age range, 4 days to 1 month) admitted to The Children's Hospital of Nanjing Medical University (Nanjing, China) between February 2018 and July 2019 were enrolled in the present study, and their plasma samples were collected prospectively. Children with immunological disorders, cardiovascular diseases and other congenital digestive tract abnormalities were excluded, as these pathological factors can affect serum miRNA levels. Plasma samples were collected in a plasma separator tube prior to any treatment and stored at $-80^{\circ} \mathrm{C}$ until use. The study was approved by the Institutional Ethics Committee of The Children's Hospital of Nanjing Medical University (approval no. 201701025). Written informed consent was obtained from a parent of each patient.

RNA extraction. Total RNA was extracted from plasma samples using TRIzol ${ }^{\circledR}$ reagent (Invitrogen; Thermo Fisher Scientific, Inc.) according to the manufacturer's protocol. In addition, $5 \mu \mathrm{l}$ of $200 \mathrm{nM}$ cel-miR-39 (Guangzhou RiboBio Co., Ltd.) was added to each sample to serve as an external control (27). RNA concentration and quality was assessed using a NanoDrop 2000 spectrophotometer (Thermo Fisher Scientific, Inc.). RNA was stored at $-80^{\circ} \mathrm{C}$ until use.

Illumina HiSeq sequencing. A total of six plasma samples (three patients with intestinal malrotation and three controls) were used for sequencing. miRNA-seq library was constructed using NEBNext Small RNA kit (NEB, cat. no. E7300S). DNA integrity was measure using an Agilent 2100 Bioanalyzer with the Agilent DNA 1000 kit (Agilent Technologies, Inc.; cat. no. 5067-1504). Sequencing was performed on the Illumina HiseqX platform (Illumina, Inc.; 150 cycles; paired-end). HiSeq X Ten Reagent kit v2.5 (Illumina, Inc.; cat. no. FC-501-25) was used for sequencing. The concentration of DNA was 30-40 nM, which was measured by real-time PCR. Ligation of 5' and 3' adaptors was carried out following manufacturer's instructions. Small RNAs were amplified by PCR (17 cycles) using adaptor-specific primers according to the manufacturer's protocol The PCR products (fragments of $\sim 90 \mathrm{bp}$ ) were purified by $8 \%$ PAGE. The size distribution of molecules in each sample were evaluated using an Agilent 2100 Bioanalyzer. Subsequently, amplification of the eligible RNAs was conducted to generate the cluster on the flow cell, which was then sequenced using the HiSeq 2000 System (single end; Illumina, Inc.), following the manufacturer's instructions. miRNAs were considered to be significantly differentially expressed if $\mathrm{P}<0.05$ and $\log _{2}$ (fold-change) $\geq 8$ for upregulated miRNAs, or $\log _{2}$ (fold-change) $\leq-8$ for downregulated miRNAs in the intestinal malrotation group, compared with the control group. These criteria applied to the average of the three samples.

Gene Ontology (GO) and Kyoto Encyclopedia of Genes and Genomes (KEGG) pathway enrichment analysis. GO functional enrichment analysis (http://geneontology.org/docs/ go-enrichment-analysis/) and KEGG pathway enrichment analysis (http://www.genome.jp/kegg/pathway.html) of DEGs were performed using DIANA TOOLS MirPath version 3 (http://diana.imis.athena-innovation.gr/DianaTools/index.php). GO has three functional categories: Molecular function (MF); biological process (BP); and cellular component (CC). $\mathrm{P}<0.05$ was considered as a statistically significant difference.

Quantification of plasma miRNA levels using reverse transcription-quantitative PCR (RT- $q P C R)$. Total RNA extracted from 20 plasma samples (10 patients with intestinal malrotation and 10 controls) was reverse transcribed to cDNA using the PrimeScript ${ }^{\mathrm{TM}}$ RT reagent kit (Takara Bio, Inc.), according to the manufacturer's instructions. RT-qPCRs were carried out using SYBR Premix Ex Taq (Takara Biotechnology Co., Ltd.) on a Light Cycler 480 (Roche Diagnostics) to measure miRNA expression levels. A total of 18 miRNA candidates (15 upregulated and three downregulated miRNAs in intestinal malrotation) were selected for RT-qPCR analysis. Thermocycling conditions consisted of an initial denaturation at $95^{\circ} \mathrm{C}$ for $10 \mathrm{~min}$, followed by 40 cycles at $94^{\circ} \mathrm{C}$ for $15 \mathrm{sec}$ and $60^{\circ} \mathrm{C}$ for $1 \mathrm{~min}$. Relative expression values were normalized against the spike-in cel-miR-39 and analyzed using the $2^{-\Delta \Delta \mathrm{Cq}}$ method (28).

Statistical analysis. Statistical analysis was carried out using GraphPad Prism 8.0 (GraphPad Software, Inc.) and SPSS 23.0 (IBM Corp.). A two-sided unpaired t-test was used to compare differences in miRNA expression between the control and intestinal malrotation groups. Receiver operating curve (ROC) analysis was performed to determine the area under the curve (AUC) of miRNA expression. SPSS 23.0 (IBM Corp.) was used to construct and analyze the curves. The final results were illustrated using GraphPad Prism 8.0 (GraphPad Software, Inc.). $\mathrm{P}<0.05$ was considered to indicate a statistically significant difference.

\section{Results}

Identification of differentially expressed serum miRNAs in intestinal malrotation. Serum miRNA levels were measured in six plasma samples (three patients with intestinal malrotation and three controls) using high-throughput sequencing. Overall, $\sim 2,588$ miRNAs were identified. The profiles of differentially expressed plasma miRNAs are presented as a heat map and a volcano plot in Fig. 1. Both the heat map and volcano plot show that patients with intestinal malrotation and healthy controls displayed markedly different plasma miRNA profiles. A total of 28 miRNAs exhibited significant differential expression, of which 21 were upregulated and 7 down regulated in patients with IM, compared with controls (Table I). 

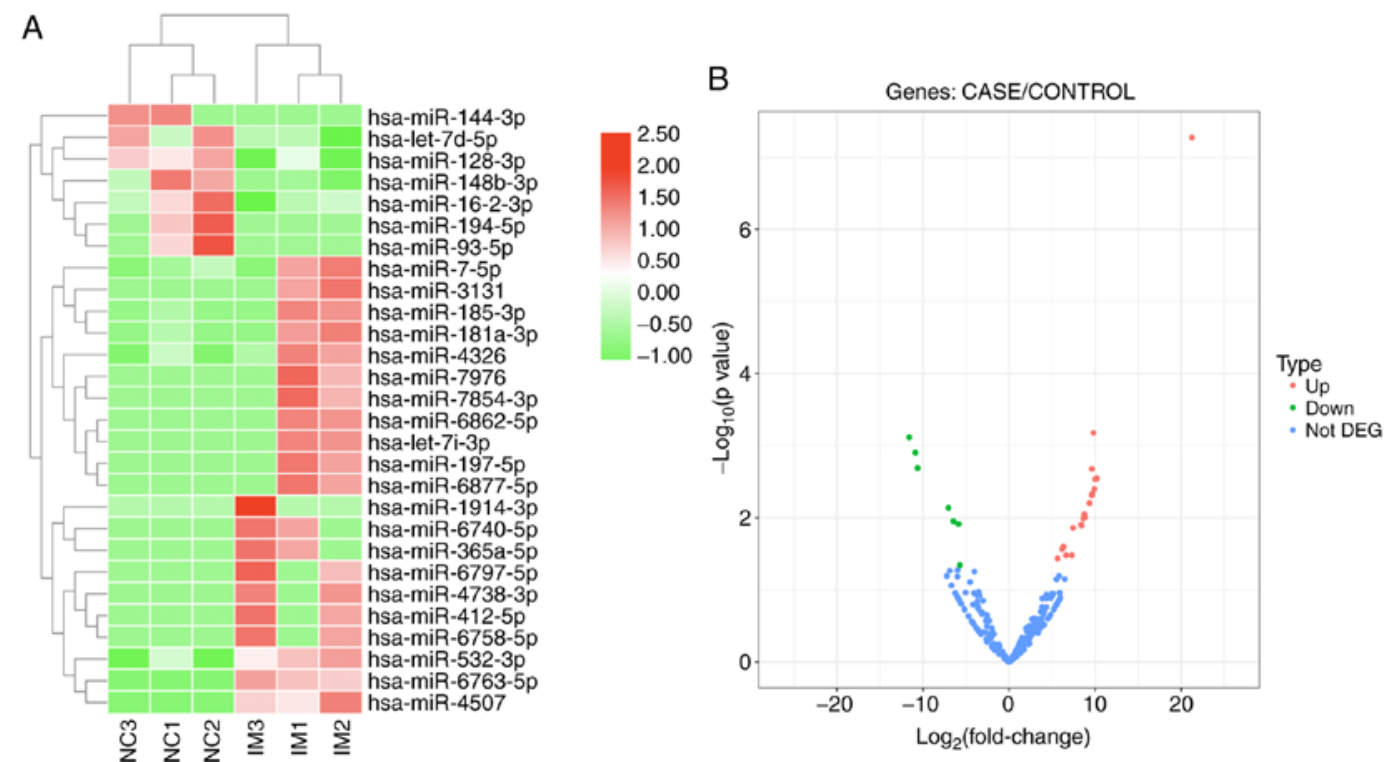

Figure 1. Heat map and volcano plot of significantly dysregulated miRNAs. (A) Heat map and (B) volcano plot of significantly dysregulated miRNAs from the serum of patients with IM. Red indicates upregulated genes and green downregulated genes with $\mathrm{P}<0.05$. miR/miRNA, microRNA; NC, negative control; IM, intestinal malrotation; DEG, differentially expressed gene; Up, upregulated; Down, downregulated.

A
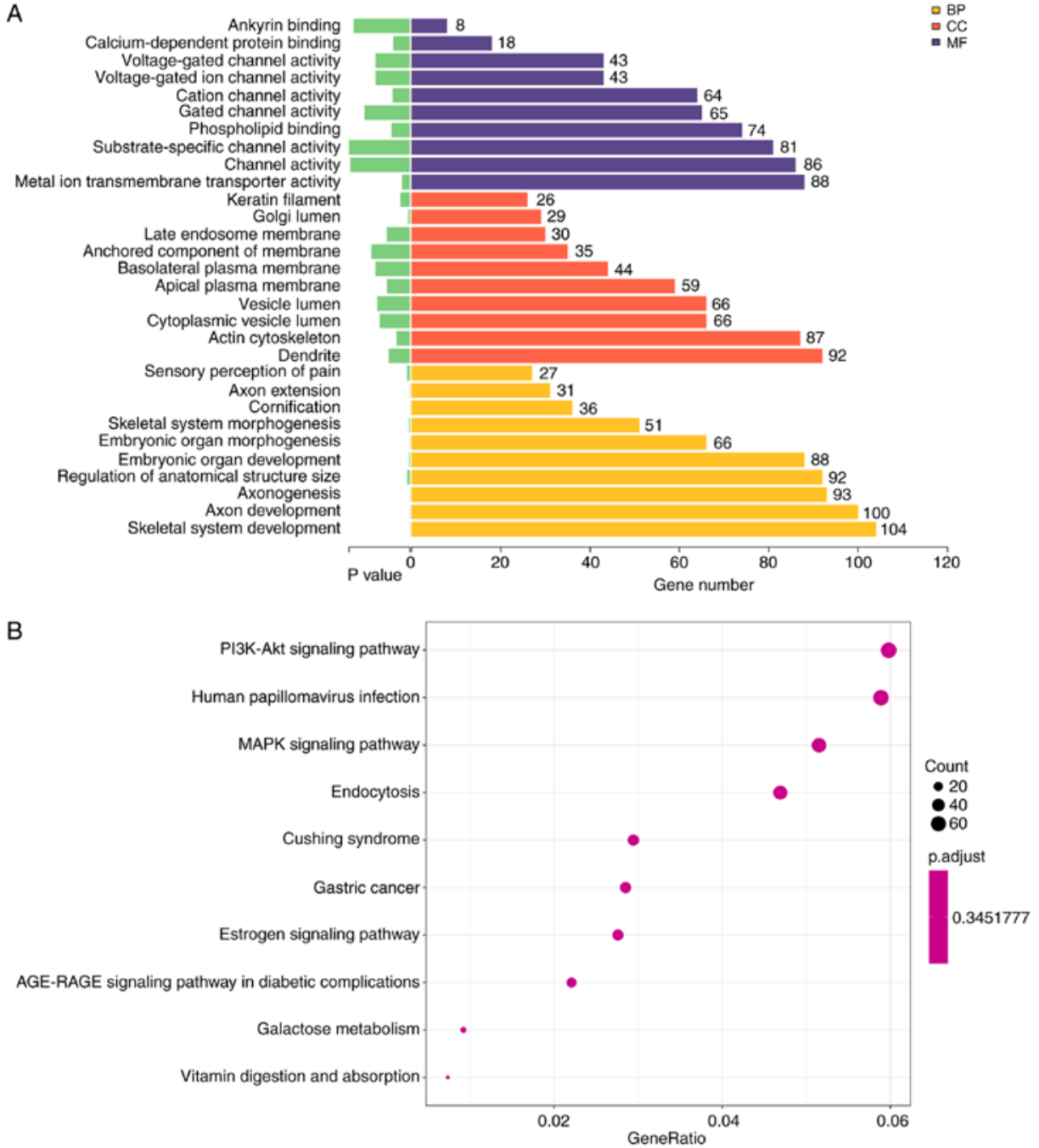

Figure 2. Bioinformatics analysis of the sequencing data. (A) GO enrichment analysis of BPs, CCs and MFs. P-values and the number of genes under each term are shown on the x-axis. GO term descriptions are presented along the y-axis. (B) Bubble graph of Kyoto Encyclopedia of Genes and Genomes enrichment results. The gene ratio of each pathway is indicated on the x-axis. Number of genes involved in each pathway proportional to the size of the bubbles. The color of the bubbles represent the P-values. GO, Gene Ontology; CC, cellular component; BP, biological process; MF, molecular function. 
Table I. Dysregulated miRNAs in plasma from patients with intestinal malrotation and controls.

\begin{tabular}{|c|c|c|c|}
\hline $\operatorname{miR}$ & $\log _{2}(\mathrm{FC})$ & P-value & Expression \\
\hline hsa-miR-1914-3p & 21.2605 & 0.00053635 & Upregulated \\
\hline hsa-miR-6763-5p & 9.8136 & 0.00066362 & Upregulated \\
\hline hsa-miR-194-5p & -11.6088 & 0.00076538 & Downregulated \\
\hline hsa-miR-144-3p & -10.8903 & 0.00124557 & Downregulated \\
\hline hsa-miR-93-5p & -10.6228 & 0.00204004 & Downregulated \\
\hline hsa-miR-185-3p & 9.6254 & 0.00209648 & Upregulated \\
\hline hsa-miR-4738-3p & 10.1860 & 0.00284375 & Upregulated \\
\hline hsa-miR-3131 & 10.0151 & 0.00290713 & Upregulated \\
\hline hsa-miR-6797-5p & 9.9124 & 0.00399543 & Upregulated \\
\hline hsa-miR-6740-5p & 9.6671 & 0.00463324 & Upregulated \\
\hline hsa-miR-412-5p & 9.6234 & 0.00487532 & Upregulated \\
\hline hsa-miR-6758-5p & 9.3378 & 0.00625270 & Upregulated \\
\hline hsa-miR-16-2-3p & -7.0390 & 0.00726665 & Downregulated \\
\hline hsa-miR-6862-5p & 8.7506 & 0.00897906 & Upregulated \\
\hline hsa-miR-365a-5p & 8.8099 & 0.00986631 & Upregulated \\
\hline hsa-miR-197-5p & 8.6241 & 0.01019434 & Upregulated \\
\hline hsa-let-7d-5p & -6.4675 & 0.01114899 & Downregulated \\
\hline hsa-miR-148b-3p & -5.8861 & 0.01219763 & Downregulated \\
\hline hsa-miR-6877-5p & 8.3920 & 0.01254641 & Upregulated \\
\hline hsa-let-7i-3p & 8.3746 & 0.01255295 & Upregulated \\
\hline hsa-miR-7976 & 8.4149 & 0.01268761 & Upregulated \\
\hline hsa-miR-4507 & 7.4372 & 0.01375498 & Upregulated \\
\hline hsa-miR-7-5p & 6.3143 & 0.02523047 & Upregulated \\
\hline hsa-miR-4326 & 6.1695 & 0.02722018 & Upregulated \\
\hline hsa-miR-181a-3p & 6.6420 & 0.03291677 & Upregulated \\
\hline hsa-miR-7854-3p & 7.2962 & 0.03293265 & Upregulated \\
\hline hsa-miR-532-3p & 5.6365 & 0.03647876 & Upregulated \\
\hline hsa-miR-128-3p & -5.7314 & 0.04537403 & Downregulated \\
\hline
\end{tabular}

miR, microRNA; FC, fold-change.

Gene Ontology and Kyoto Encyclopedia of Genes and Genomes (KEGG) pathway enrichment analysis. GO analysis suggested that the differentially expressed miRNAs were mainly involved in involved in 'skeletal system development', 'axon development' (Fig. 2A, Table II). These miRNAs were mainly localized or exerted their functions in dendrites and the actin cytoskeleton (Fig. 2A, Table III). Moreover, these miRNAs were involved in 'metal ion transmembrane transporter activity', 'channel activity' and 'substrate-specific channel activity' and other molecular functions (Fig. 2A; Table IV).

KEGG pathway analysis identified 58 significantly enriched pathways in the sequenced miRNAs. The 10 most enriched pathway terms were 'PI3K-Akt signaling pathway', 'human papillomavirus infection', 'MAPK signaling pathway', 'endocytosis', 'Cushing syndrome', 'gastric cancer', 'estrogen signaling pathway', 'AGE-RAGE signaling pathway in diabetic complications', 'galactose metabolism' and 'vitamin digestion and absorption' (Fig. 2B). The most significant KEGG pathway was 'Endocytosis' (KEGG ID, hsa04144), which involved 51 target genes $(\mathrm{P}=001$; Table $\mathrm{V})$. The network associated was analyzed, which demonstrated TGF- $\beta$ signaling pathway and endosomal recycling compartment were mainly affected (Fig. 3). Thus, GO and KEGG analysis provided novel insights into the underlying mechanism by which plasma miRNAs might participate in the pathogenesis of intestinal malrotation.

Selection and validation of potential plasma miRNA biomarkers for intestinal malrotation. A total of 18 miRNAs were differentially expressed. Of these, 15 miRNAs were upregulated, including, hsa-miR-1914-3p, miR-6763-5p, miR-185-3p, miR-4738-3p, miR-3131, miR-6797-5p, miR-6740-5p, miR-412-5p, miR-6758-5p, miR-6862-5p, miR-365a-5p, miR-197-5p, miR-6877-5p, let-7 and miR-7976. Moreover, three were downregulated, hsa-miR-194-5p, $-144-3 p$ and $-93-5 p$ (Table I) and were selected for further validation. The expression levels of all 18 candidates were measured in a larger sample size (10 patients with intestinal malrotation and 10 controls) by RT-qPCR. In total, nine miRNAs were confirmed to be differentially expressed. Of these, six were upregulated, compared with the control group, including hsa-miR-1914-3p, -6763-5p, -185-3p, -3131, 
Table II. Top 10 significantly enriched GO biological processes.

\begin{tabular}{llrr}
\hline GO term & \multicolumn{1}{c}{ Description } & \multicolumn{1}{c}{ P-value } \\
\hline GO:0070268 & Cornification & 0.00000056 & 36 \\
GO:0001501 & Skeletal system development & 0.00000277 & 104 \\
GO:0061564 & Axon development & 0.00000467 & 100 \\
GO:0007409 & Axonogenesis & 0.00000507 & 93 \\
GO:0048562 & Embryonic organ morphogenesis & 0.00000752 & 66 \\
GO:0048675 & Axon extension & 0.00000985 & 31 \\
GO:0048705 & Skeletal system morphogenesis & 0.00002872 & 51 \\
GO:0048568 & Embryonic organ development & 0.00002935 & 88 \\
GO:0090066 & Regulation of anatomical structure size & 0.00009987 & 92 \\
GO:0019233 & Sensory perception of pain & 0.00010642
\end{tabular}

GO, Gene Ontology.

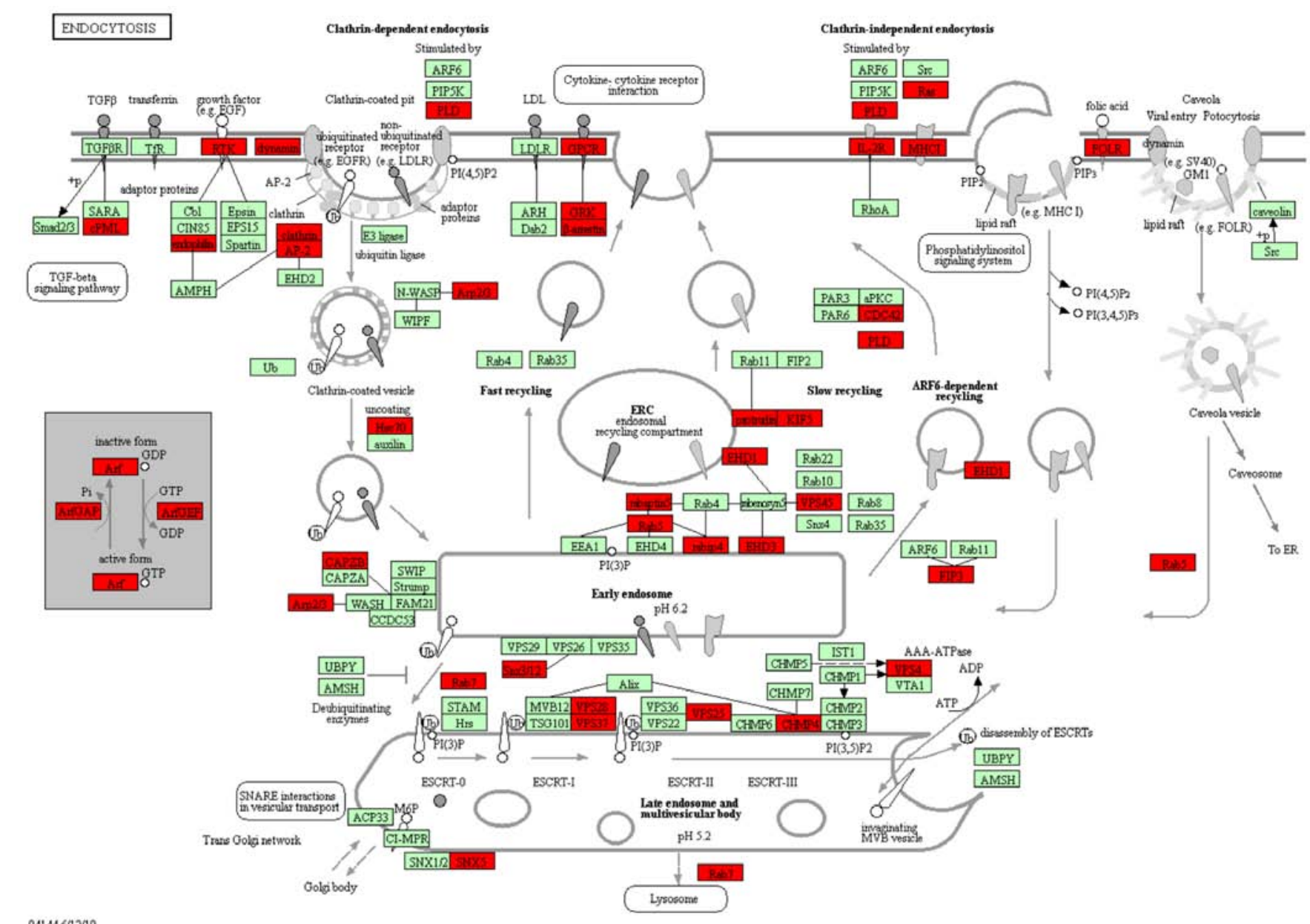

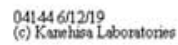

Figure 3. Detailed view of the 'endocytosis' network. Each node represents a key transporter or enzyme. The red nodes indicate miRNA with significantly expression, while the green notes represent miRNAs with no significant change in expression.

$-6740-5 p$ and $-6758-5 p$. In addition, three were downregulated, hsa-miR-194-5p, -144-3p and -93-5p (Fig. 4; Table VI).

ROC curve analysis. The sensitivity and specificity of the aforementioned 9 miRNAs as potential diagnostic biomarkers of intestinal malrotation were determined using ROC curve analysis. The AUCs of these miRNAs ranged from 0.727-0.975 (Fig. 5; Table VII). MiR-1914-3p (AUC, 0.975; 95\% CI, 0.913-1.000) and miR-3131 (AUC, 0.975; 95\% CI, 0.913-1.000) presented the largest AUCs, followed by miR-185-3p (AUC, 0.958; 95\% CI, 0.873-1.000) and miR-6763-5p (AUC, 0.950; 95\% CI, 0.856-1.000). 
Table III. Top 10 significantly enriched GO cellular components.

\begin{tabular}{llll}
\hline GO term & \multicolumn{1}{c}{ Description } & P-value & Count \\
\hline GO:0005796 & Golgi lumen & 0.00007119 & 29 \\
GO:0045095 & Keratin filament & 0.00038711 & 26 \\
GO:0015629 & Actin cytoskeleton & 0.00054521 & 87 \\
GO:0030425 & Dendrite & 0.00086110 & 92 \\
GO:0016324 & Apical plasma membrane & 0.00093914 & 59 \\
GO:0031902 & Late endosome membrane & 0.00094567 & 30 \\
GO:0060205 & Cytoplasmic vesicle lumen & 0.00123996 & 66 \\
GO:0031983 & Vesicle lumen & 0.00134049 & 66 \\
GO:0016323 & Basolateral plasma membrane & 0.00140855 & 44 \\
GO:0031225 & Anchored component of membrane & 0.00158692 & 35
\end{tabular}

GO, Gene Ontology.
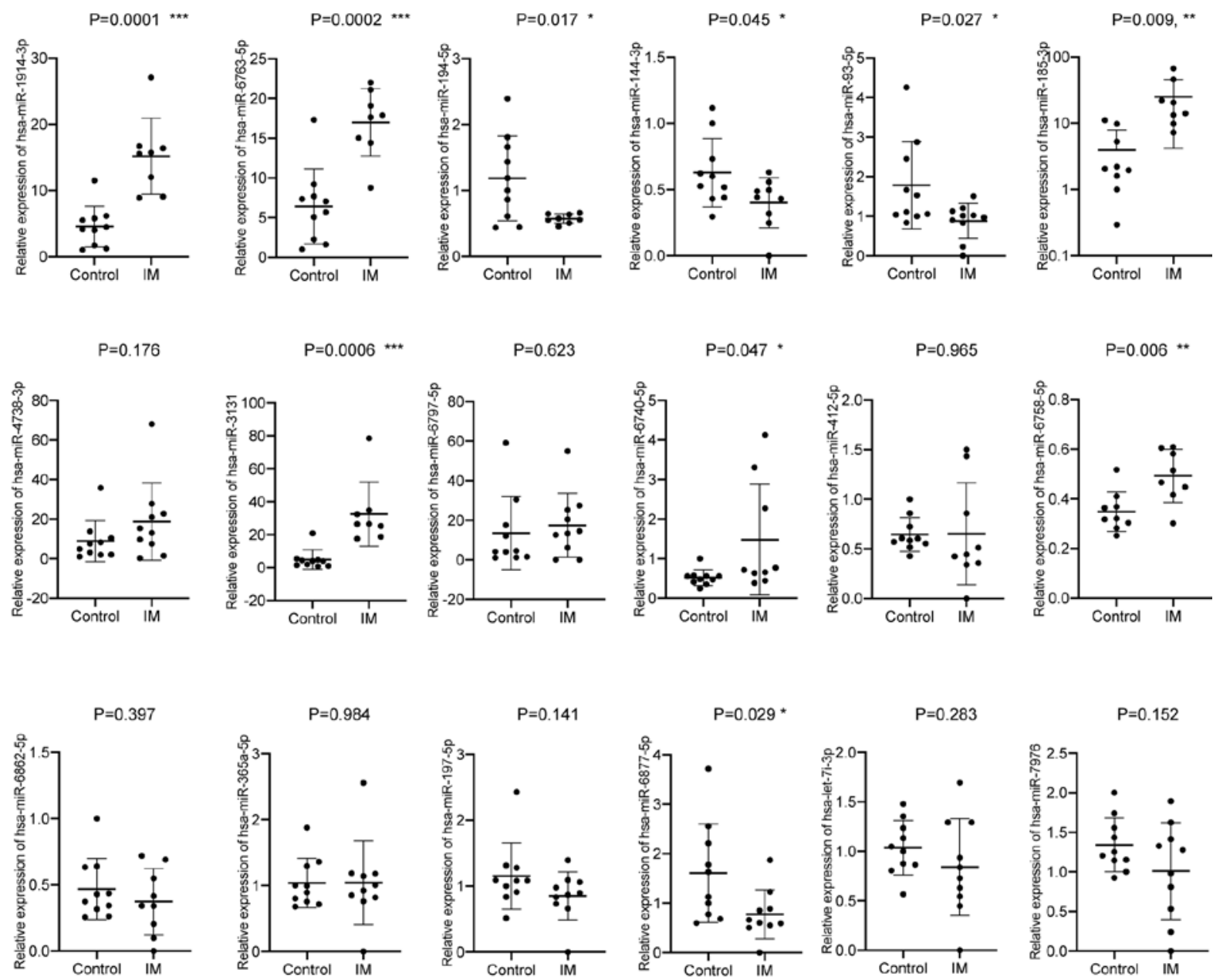

Figure 4. Expression levels of 18 candidate miRNAs in the serum of patients with IM and matched controls. miRNA/miR, microRNA; IM, intestinal malrotation. ${ }^{*} \mathrm{P}<0.05 ;{ }^{* *} \mathrm{P}<0.01,{ }^{* * *} \mathrm{P}<0.001$, vs. control.

\section{Discussion}

Malrotation is a congenital abnormal bowel position within the peritoneal cavity that usually involves both the small and the large bowel (29). The term malrotation encompasses a wide range of rotational and fixation abnormalities of the intestines, from readily apparent omphaloceles in newborns to asymptomatic non-rotation in adults (1). Congenital intestinal non-rotation and incomplete rotation usually concur with a narrow base of the mesentery, which might cause duodenal obstruction and midgut volvulus and manifest as acute symptoms (30). Pediatric patients who do not present acute symptoms but are not diagnosed before 
Table IV. Top 10 significantly enriched GO molecular functions.

\begin{tabular}{llrr}
\hline GO term & \multicolumn{1}{c}{ Description } & P-value & Count \\
\hline GO:0046873 & Metal ion transmembrane transporter activity & 0.00032224 & 88 \\
GO:0048306 & Calcium-dependent protein binding & 0.00068622 & 18 \\
GO:0005261 & Cation channel activity & 0.00070680 & 64 \\
GO:0005543 & Phospholipid binding & 0.00075808 & 74 \\
GO:0005244 & Voltage-gated ion channel activity & 0.00140047 & 43 \\
GO:0022832 & Voltage-gated channel activity & 0.00140047 & 43 \\
GO:0022836 & Gated channel activity & 0.00187551 & 85 \\
GO:0030506 & Ankyrin binding & 0.00233200 & 86 \\
GO:0015267 & Channel activity & 0.00245557 & 8.00251147
\end{tabular}

GO, Gene Ontology.
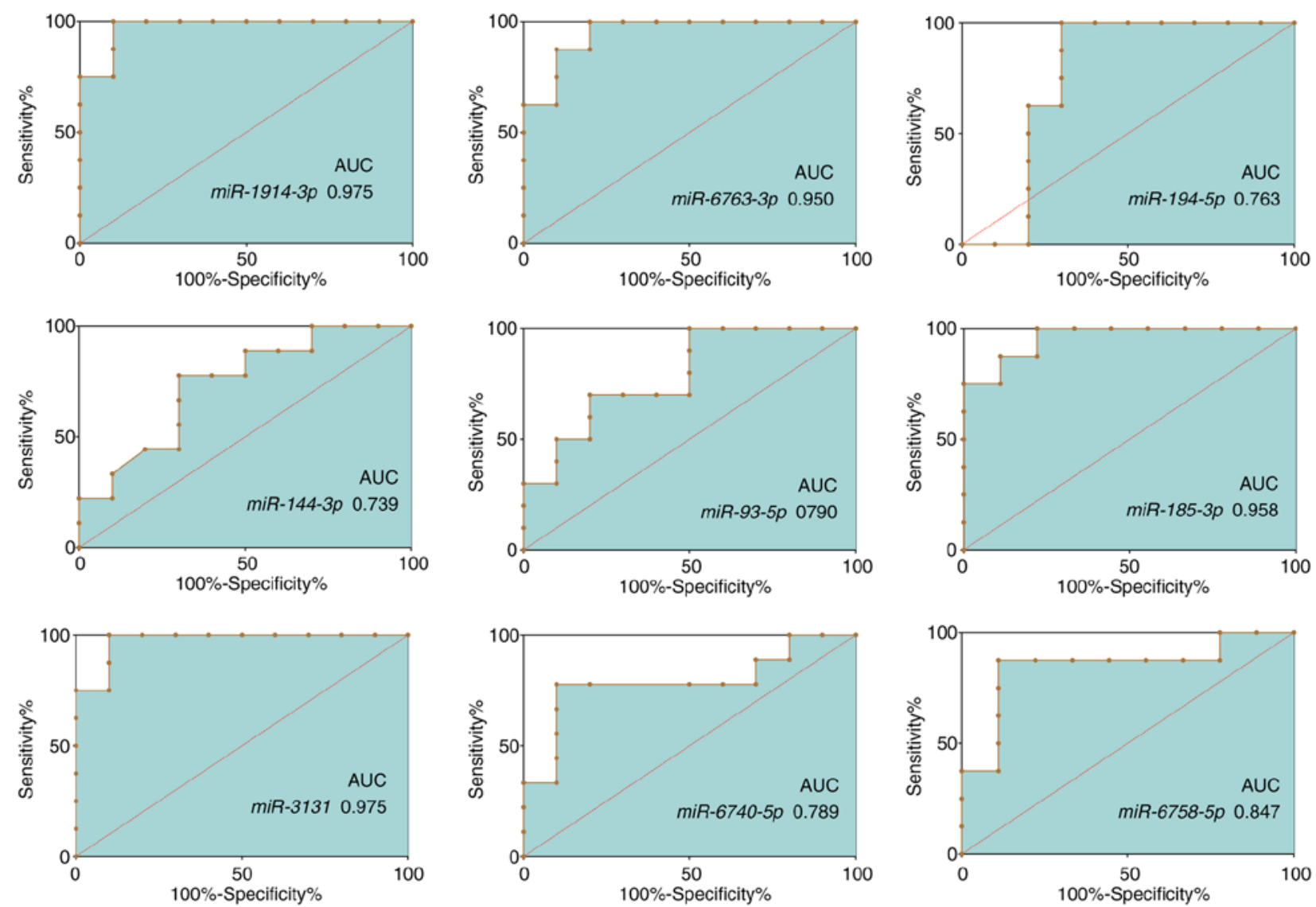

Figure 5. Receiver operating characteristic curves of selected dysregulated miRNAs. Expression of nine candidate miRNAs was assessed using reverse transcription-quantitative PCR. The corresponding AUCs are presented in the diagrams. miRNA/miR, microRNA; AUC, area under the curve.

adulthood are at risk of developing various chronic symptoms later in life, including vomiting, vague abdominal pain, diarrhea, nausea, early satiety and bloating, dyspepsia and other functional or psychiatric disorders $(31,32)$.

Approximately $90 \%$ of infants with intestinal malrotation are diagnosed within the first year of life, and $\sim 80 \%$ within the first month (33). Common diagnostic tools include UGI, ultrasonography, and barium enema. Although the duodenojejunal junction is usually fixed at the Treitz ligament and functions as a useful landmark, in some cases UGI results may be confusing or hard to interpret, and diagnosis of these cases of malrotation depends on the recognition of anatomic subtleties $(8,34)$. Both false positive and false negative findings have been reported $(7,8,35,36)$. Barium enema can help to some extent in some cases, but challenges exist in terms of the quantity of barium administered to children and the observation of the first pass of barium through the duodenum (5). Moreover, the interpretation of 
Table V. Top 10 significantly enriched KEGG pathways.

\begin{tabular}{llrr}
\hline KEGG pathway & \multicolumn{1}{c}{ Description } & P-value & Count \\
\hline hsa04144 & Endocytosis & 0.00132301 & 51 \\
hsa05165 & Human papillomavirus infection & 0.00253633 & 64 \\
hsa04933 & AGE-RAGE signaling pathway in diabetic complications & 0.00411644 & 24 \\
hsa04915 & Estrogen signaling pathway & 0.00688242 & 30 \\
hsa00052 & Galactose metabolism & 0.00689000 & 10 \\
hsa04010 & MAPK signaling pathway & 0.00724133 & 56 \\
hsa04151 & PI3K-Akt signaling pathway & 0.00865405 & 65 \\
hsa05226 & Gastric cancer & 0.01159650 & 31 \\
hsa04934 & Cushing syndrome & 0.01168848 & 32 \\
hsa04977 & Vitamin digestion and absorption & 0.01228429 & 8 \\
\hline
\end{tabular}

KEGG, Kyoto Encyclopedia of Genes and Genomes.

Table VI. Relative expression of nine candidate miRNAs validated by reverse transcription-quantitative PCR from patients with IM and controls.

\begin{tabular}{lcccc}
\hline miRNA & Controls & Patients with IM & P-value & Fold change \\
\hline miR-1914-3p & 4.552 & 15.17 & $0.0001^{\mathrm{c}}$ & 3.333 \\
miR-6763-5p & 6.422 & 16.99 & $0.0002^{\mathrm{c}}$ & 2.646 \\
miR-185-3p & 3.923 & 25.02 & $0.0092^{\mathrm{b}}$ & 6.378 \\
miR-3131 & 4.868 & 32.54 & $0.0006^{\mathrm{c}}$ & 6.684 \\
miR-194-5p & 1.183 & 0.571 & $0.0170^{\mathrm{a}}$ & 0.483 \\
miR-6740-5p & 0.522 & 1.479 & $0.0470^{\mathrm{a}}$ & 2.833 \\
miR-144-3p & 0.628 & 0.401 & $0.0449^{\mathrm{a}}$ & 0.639 \\
miR-6758-5p & 0.349 & 0.493 & $0.0061^{\mathrm{b}}$ & 1.413 \\
miR-93-5p & 1.783 & 0.881 & $0.0272^{\mathrm{a}}$ & 0.494 \\
\hline
\end{tabular}

${ }^{\mathrm{a}} \mathrm{P}<0.05 ;{ }^{\mathrm{b}} \mathrm{P}<0.01,{ }^{\mathrm{c}} \mathrm{P}<0.001$ vs. control. miR/miRNA. microRNA; IM, intestinal malrotation. The values in controls and Patients with IM columns represent the relative expression values.

Table VII. The respective AUCs of nine candidate miRNAs.

\begin{tabular}{lcccc}
\hline miRNA & AUC & Standard error & Asymptotic significance & Asymptotic 95\% CI \\
\hline miR-1914-3p & 0.975 & 0.032 & $<0.001$ & $0.913-1.000$ \\
miR-6763-5p & 0.950 & 0.048 & 0.001 & $0.856-1.000$ \\
miR-185-3p & 0.958 & 0.044 & 0.002 & $0.873-1.000$ \\
miR-3131 & 0.975 & 0.032 & $<0.001$ & $0.913-1.000$ \\
miR-194-5p & 0.763 & 0.128 & 0.062 & $0.512-1.000$ \\
miR-6740-5p & 0.789 & 0.115 & 0.034 & $0.564-1.000$ \\
miR-144-3p & 0.739 & 0.116 & 0.079 & $0.512-0.966$ \\
miR-6758-5p & 0.847 & 0.106 & 0.016 & $0.639-1.000$ \\
miR-93-5p & 0.790 & 0.102 & 0.028 & $0.590-0.991$ \\
\hline
\end{tabular}

$\mathrm{miR} / \mathrm{miRNA}$, microRNA; AUC, area under the curve.

ultrasonographical findings can be very subjective, and the presence of an experienced radiologist or technician, who may not always be available at every hospital or medical center, is essential (10). 
In the present study, potential diagnostic biomarkers were identified by analyzing plasma miRNA levels in pediatric patients with intestinal malrotation. A profile of differentially expressed miRNAs in the plasma of intestinal malrotation patients was obtained by sequencing plasma miRNAs, and a number of differentially expressed miRNAs were identified, including 21 upregulated and seven downregulated miRNAs. Subsequently, bioinformatics analyses were carried out to investigate the potential involvement of differentially expressed miRNAs in intestinal malrotation.

GO enrichment analysis suggested that these miRNAs were mainly involved in 'metal ion transmembrane transporter activity', 'calcium-dependent protein binding', 'cation channel activity' and 'phospholipid binding'. 'Metal ion transmembrane transporter activity' and 'calcium-dependent protein binding' were the most significantly enriched GO terms with respect to molecular function. Interestingly, it was previously reported that calcium channel-blocking drugs could lead to many developmental abnormalities in Xenopus embryos, including malrotation of the gut, possibly through dysregulation of calcium ion antagonism (37). However, the potential link between plasma miRNA levels and calcium ion antagonism in intestinal malrotation requires further study to determine where and how plasma miRNAs interfere with calcium ion. 'Cornification' was the most significantly enriched GO term with respect to biological processes, followed by 'skeletal system development cornification'. A previous study reported a higher frequency of malformations in the skeleton system, including the facial skeleton, the small pelvis, and the upper and lower extremities, among pediatric patients with intestinal malrotation (38). The present results indicated that plasma miRNAs might play a role in this association. The most enriched KEGG pathway was 'endocytosis', which is a critical process in the intestine that is responsible for the endocytic uptake of macromolecules from the gut lumen. For instance, but its role in intestinal malrotation has not yet been studied $(39,40)$.

miRNAs have been demonstrated to play a variety of roles in numerous intestinal diseases and several could even function as biomarkers (41-43). Another important finding of the present study was that plasma miRNAs have the potential to serve as circulating biomarkers for intestinal malrotation. Based on the sequencing data and validation by RT-qPCR in a larger number of samples, nine miRNAs were differentially expressed, including six upregulated miRNAs (hsa-miR-1914-3p, -6763-5p, -185-3p, -3131, -6740-5p and $-6758-5 p$ ) and three downregulated miRNAs (hsa-miR-194-5p, $-144-3 p$ and $-93-5 p)$. The highest AUCs were obtained for miR-1914-3p, -3131, -185-3p and -6763-5p, suggesting that these miRNAs may serve as diagnostic biomarkers for intestinal malrotation. As a following step, studies with larger sample sizes are necessary to validate the present findings.

In conclusion, a profile of differentially expressed miRNAs in the plasma of patients with intestinal malrotation was identified, and the potential molecular functions associated with these miRNAs were described. Four of the nine confirmed differentially expressed miRNAs have the potential to improve early diagnosis of intestinal malrotation. However, these miRNAs exhibited relatively small $95 \%$ CIs, which is a great limitation to our study and requires further confirmation in the future with much larger sample size. These results also suggested that plasma miRNAs play an important role in intestinal malrotation, although this hypothesis requires further investigation.

\section{Acknowledgements}

Not applicable.

\section{Funding}

This work was funded by The Natural Science Foundation of China (grant no. NSFC 81701493) and The Nanjing Science and Technology Development Project (grant no. 201723006).

\section{Availability of data and materials}

The datasets used and/or analyzed during the current study are available from the corresponding author on reasonable request.

\section{Authors' contributions}

XL, HL and CL designed the study. HC and XS collected the samples and conducted the sequencing. $\mathrm{XL}$ and $\mathrm{LZ}$ carried out the PCRs and ROC analysis. XL contributed to writing the manuscript. All authors read and approved the final manuscript.

\section{Ethics approval and consent to participate}

This study was approved by The Institutional Ethics Committee of The Children's Hospital of Nanjing Medical University (approval no. 201701025). Written informed consent was obtained from a parent of each enrolled patient.

\section{Patient consent for publication}

Not applicable.

\section{Competing interests}

The authors declare that they have no competing interests.

\section{References}

1. Applegate KE, Anderson JM and Klatte EC: Intestinal malrotation in children: A problem-solving approach to the upper gastrointestinal series. Radiographics 26: 1485-1500, 2006.

2. Blumberg K: Intestinal malrotation. Radiology 202: 584, 1997.

3. Coste AH, Waheed A and Ahmad H: Midgut Volvulus. StatPearls Publishing, Treasure Island, FL, 2019.

4. Adams SD and Stanton MP: Malrotation and intestinal atresias. Early Hum Dev 90: 921-925, 2014.

5. Morris G, Kennedy A Jr and Cochran W: Small bowel congenital anomalies: A review and update. Curr Gastroenterol Rep 18: 16, 2016.

6. Graziano K, Islam S, Dasgupta R, Lopez ME, Austin M, Chen LE, Goldin A, Downard CD, Renaud E and Abdullah F: Asymptomatic malrotation: Diagnosis and surgical management: An American Pediatric Surgical Association outcomes and evidence based practice committee systematic review. J Pediatr Surg 50: 1783-1790, 2015.

7. Dilley AV, Pereira J, Shi EC, Adams S, Kern IB, Currie B and Henry GM: The radiologist says malrotation: Does the surgeon operate? Pediatr Surg Int 16: 45-49, 2000 
8. Long FR, Kramer SS, Markowitz RI, Taylor GE and Liacouras CA Intestinal malrotation in children: Tutorial on radiographic diagnosis in difficult cases. Radiology 198: 775-780, 1996.

9. Strouse PJ: Malrotation. Semin Roentgenol 43: 7-14, 2008.

10. Taylor GA: CT appearance of the duodenum and mesenteric vessels in children with normal and abnormal bowel rotation. Pediatr Radiol 41: 1378-1383, 2011.

11. Tackett JJ, Muise ED and Cowles RA: Malrotation: Current strategies navigating the radiologic diagnosis of a surgical emergency. World J Radiol 6: 730-736, 2014.

12. Dekonenko C, Sujka JA, Weaver K, Sharp SW, Gonzalez K and St Peter SD: The identification and treatment of intestinal malrotation in older children. Pediatr Surg Int 35: 665-671, 2019.

13. Kroh EM, Parkin RK, Mitchell PS and Tewari M: Analysis of circulating microRNA biomarkers in plasma and serum using quantitative reverse transcription-PCR (qRT-PCR). Methods 50: 298-301, 2010.

14. Patel M, Verma A, Aslam I, Pringle H and Singh B: Novel plasma microRNA biomarkers for the identification of colitis-associated carcinoma. Lancet 385 (Suppl 1): S78, 2015.

15. van de Vrie M, Deegens JK, Eikmans M, van der Vlag J and Hilbrands LB: Urinary MicroRNA as biomarker in renal transplantation. Am J Transplant 17: 1160-1166, 2017.

16. Panico C and Condorelli G: microRNA-132: A new biomarker of heart failure at last? Eur J Heart Fail 20: 86-88, 2018.

17. Liu R, Chen X, Du Y, Yao W, Shen L, Wang C, Hu Z, Zhuang R, Ning G, Zhang C, et al: Serum microRNA expression profile as a biomarker in the diagnosis and prognosis of pancreatic cancer. Clin Chem 58: 610-618, 2012.

18. McGuire A, Brown JA and Kerin MJ: Metastatic breast cancer: The potential of miRNA for diagnosis and treatment monitoring. Cancer Metastasis Rev 34: 145-155, 2015.

19. Fabris L, Ceder Y, Chinnaiyan AM, Jenster GW, Sorensen KD Tomlins S, Visakorpi $\mathrm{T}$ and Calin GA: The potential of microRNAs as prostate cancer biomarkers. Eur Urol 70: 312-322, 2016.

20. Zampetaki A, Willeit P, Tilling L, Drozdov I, Prokopi M, Renard JM, Mayr A, Weger S, Schett G, Shah A, et al: Prospective study on circulating microRNAs and risk of myocardial infarction. J Am Coll Cardiol 60: 290-299, 2012.

21. Barwari T, Joshi A and Mayr M: MicroRNAs in cardiovascular disease. J Am Coll Cardiol 68: 2577-2584, 2016.

22. Lu TX and Rothenberg ME: MicroRNA. J Allergy Clin Immunol 141: 1202-1207, 2018.

23. Kumar S, Vijayan M, Bhatti JS and Reddy PH: MicroRNAs as peripheral biomarkers in aging and age-related diseases. Prog Mol Biol Transl Sci 146: 47-94, 2017.

24. Backes C, Meese E and Keller A: Specific miRNA disease biomarkers in blood, serum and plasma: Challenges and prospects. Mol Diagn Ther 20: 509-518, 2016.

25. Miranda J, Rocha G, Soares P, Morgado H, Baptista MJ, Azevedo I, Fernandes S, Brandão O, Sen P and Guimarães H: A novel mutation in FOXF1 gene associated with alveolar capillary dysplasia with misalignment of pulmonary veins, intestinal malrotation and annular pancreas. Neonatology 103: 241-245, 2013.

26. Martin V and Shaw-Smith C: Review of genetic factors in intestinal malrotation. Pediatr Surg Int 26: 769-781, 2010.
27. Sohn W, Kim J, Kang SH, Yang SR, Cho JY, Cho HC, Shim SG and Paik YH: Serum exosomal microRNAs as novel biomarkers for hepatocellular carcinoma. Exp Mol Med 47: e184, 2015.

28. Livak KJ and Schmittgen TD: Analysis of relative gene expression data using real-time quantitative PCR and the 2(-Delta Delta C(T)) Method. Methods 25: 402-408, 2001.

29. Powell DM, Othersen HB and Smith CD: Malrotation of the intestines in children: The effect of age on presentation and therapy. J Pediatr Surg 24: 777-780, 1989.

30. Gross E, Chen MK and Lobe TE: Laparoscopic evaluation and treatment of intestinal malrotation in infants. Surg Endosc 10: 936-937, 1996.

31. Durkin ET, Lund DP, Shaaban AF, Schurr MJ and Weber SM: Age-related differences in diagnosis and morbidity of intestinal malrotation. J Am Coll Surg 206: 658-663, 2008.

32. Lin JN, Lou CC and Wang KL: Intestinal malrotation and midgut volvulus: A 15-year review. J Formos Med Assoc 94: 178-181, 1995.

33. Hwang SM, Na YS, Cho Y, You DG and Lee JJ: Midgut volvulus as a complication of intestinal malrotation in a term pregnancy. Korean J Anesthesiol 67 (Suppl): S98-S99, 2014.

34. Beasley SW and de Campo JF: Pitfalls in the radiological diagnosis of malrotation. Australas Radiol 31: 376-383, 1987.

35. Prasil P, Flageole H, Shaw KS, Nguyen LT, Youssef S and Laberge JM: Should malrotation in children be treated differently according to age? J Pediatr Surg 35: 756-758, 2000.

36. Torres AM and Ziegler MM: Malrotation of the intestine. World J Surg 17: 326-331, 1993.

37. Burgess AM and Vere DW: Teratogenic effects of some calcium channel blocking agents in Xenopus embryos. Pharmacol Toxicol 64: 78-82, 1989.

38. Botvin'ev OK, Eremeeva AV, Razumovskaia IN and Kondrikova EV: Intestinal malrotation: Genetics features and other congenital malformations in children. Arkh Patol 73: 29-32, 2011 (In Russian).

39. Haq S, Grondin J, Banskota S and Khan WI: Autophagy: Roles in intestinal mucosal homeostasis and inflammation. J Biomed Sci 26: 19, 2019.

40. Bujko A,Atlasy N,Landsverk OJB,RichterL, YaqubS,HornelandR, Øyen O, Aandahl EM, Aabakken L, Stunnenberg HG, et al: Transcriptional and functional profiling defines human small intestinal macrophage subsets. J Exp Med 215: 441-458, 2018.

41. Yoshikawa T, Wu J, Otsuka M, Kishikawa T, Suzuki N, Takata A, Ohno M, Ishibashi R, Yamagami M, Nakagawa R, et al: Repression of microRNA function mediates inflammation-associated colon tumorigenesis. Gastroenterology 152: 631-643, 2017.

42. Xiao L, Wu J, Wang JY, Chung HK, Kalakonda S, Rao JN, Gorospe $\mathrm{M}$ and Wang JY: Long noncoding RNA uc. 173 promotes renewal of the intestinal mucosa by inducing degradation of microRNA 195. Gastroenterology 154: 599-611, 2018

43. Cao B, Zhou X, Ma J, Zhou W, Yang W, Fan D and Hong L: Role of miRNAs in inflammatory bowel disease. Dig Dis Sci 62: 1426-1438, 2017. International (CC BY-NC-ND 4.0) License. 